Received: 29 March 2017

Accepted: 19 June 2017

Published online: 25 July 2017
Solid-State ${ }^{77}$ Se NMR of

\section{Organoselenium Compounds} through Cross Polarization Magic Angle Spinning (CPMAS) Method

Duo Wei ${ }^{1}$, Mengting $\mathrm{Han}^{2}$ \& LeiYu

Characterization of selenium states by ${ }^{77}$ Se NMR is quite important to provide vital information for mechanism studies in organoselenium-catalyzed reactions. With the development of heterogeneous polymer-supported organoselenium catalysts, the solid state ${ }^{77} \mathrm{Se} N \mathrm{NR}$ comes to the spotlight. It is necessary to figure out an advanced protocol that provides good quality spectra within limited time because solid state ${ }^{77}$ Se NMR measurements are always time consuming due to the long relaxation time and the relatively low sensitivity. Studies on small molecules and several novel polymer-supported organoselenium materials in this article showed that cross polarization (CP) method with the assistance of magic angle spinning (MAS) was more efficient to get high quality spectra than the methods by using single pulse (SP) or high power ${ }^{1} \mathrm{H}$ decoupling (HPHD) combined with MAS. These results lead to a good understanding of the effect of the molecular structure, the heteronuclear coupling, the long-range ordering of the solid (crystal or amorphous), and the symmetry of ${ }^{77}$ Se on quality of their spectra.

Selenium is a rare element first discovered by Swedish chemist Berzelius in 1818. It has been incorporated in nearly all areas of chemistry now because of the unique chemical and biological properties ${ }^{1-6}$. Recently, the catalytic activities of organoselenium compounds have attracted much attention ${ }^{7-34}$. Because selenium is a metabolizable element that will not accumulate in the body ${ }^{35}$, organoselenium catalysts are much safer for both organisms and the environment than transition metal catalysts. Organoselenium-catalyzed reactions usually employed hydrogen peroxide as the clean oxidant that generated no wastes other than the water ${ }^{20-34}$. Organoselenium catalysts were very stable and could be recycled and reused for many times without deactivation ${ }^{24-34}$. Binding onto polymers, the heterogeneous organoselenium catalysts were even easier to be separated from the reaction solutions and were of profound industrial application values ${ }^{25}$. During our continuous investigations on organoselenium catalysis ${ }^{24-34}$, we found that the selectivity of organoselenium-catalyzed reactions was tunable and different organoselenium catalysts might lead to different reaction paths ${ }^{28}$. Mechanism studies were very important to understand the protean reaction selectivity.

Besides control experiments, ${ }^{77} \mathrm{Se}$ NMR tests provided direct information of selenium states in reactions and were very efficient tools for mechanism study ${ }^{24-27}$. It has a wide chemical shift over $6000 \mathrm{ppm}$, which is beneficial for producing sufficient resolution to separate signals from different chemical sites ${ }^{36,37}$. However, ${ }^{77} \mathrm{Se}$ has a low natural abundance (ca. 7.63\%) and low relative NMR sensitivity $\left(6.93 \times 10^{-3}\right)^{36-38}$. The situation gets worse for solid-state NMR (SSNMR) especially when the Se content is even low and more complicated factors are involved. It is well known that the line width in SSNMR spectrum is usually wider than that in solution NMR because of the strong chemical shift anisotropy (CSA), dipolar-dipolar coupling, etc. Magic-angle spinning (MAS) is used routinely in the majority of SSNMR to remove the effects of CSA, to assist in the removal of heteronuclear dipolar-coupling effects, and to narrow lines from quadrupolar nuclei. Traditional single pulse (SP) sequence is always applied with the help of MAS, but still leads to low signal-noise ratio (SNR) for the characterization of ${ }^{77} \mathrm{Se}$.

The aim of the present article is to find out factors that affect the solid NMR spectrum and the best protocol to get high quality spectrum for several novel selenium-contained polymers. A method by using a combination of cross-polarization (CP) and MAS was applied, which has been previously used for the characterization of ${ }^{13} \mathrm{C},{ }^{31} \mathrm{P}$

${ }^{1}$ Testing Center, Yangzhou University, Yangzhou, 225008, China. ${ }^{2}$ School of Chemistry and Chemical Engineering, Yangzhou University, Yangzhou, 225002, China. Correspondence and requests for materials should be addressed to D.W. (email: weiduo@yzu.edu.cn) or L.Y. (email: yulei@yzu.edu.cn) 
(e)
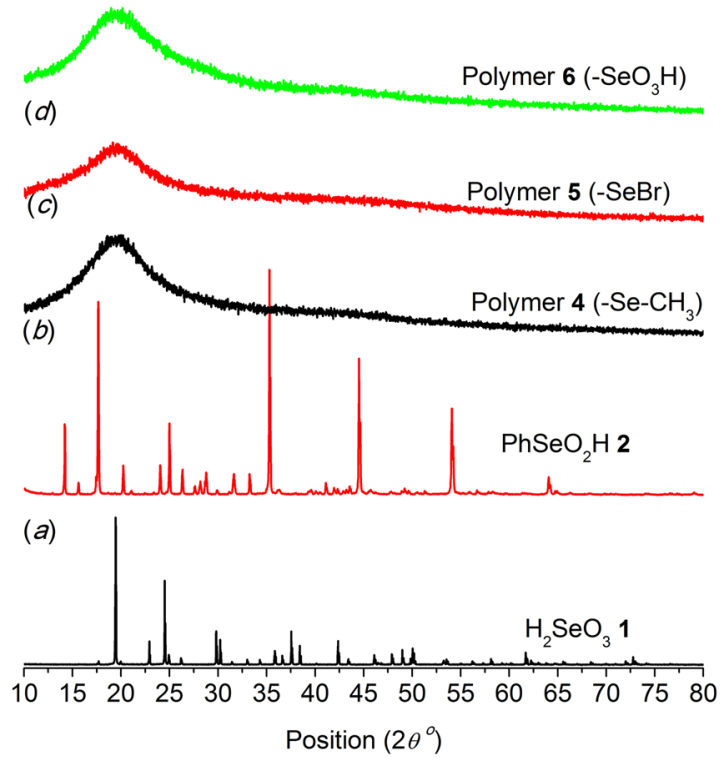

Figure 1. PXRD patterns: (a) $\mathrm{H}_{2} \mathrm{SeO}_{3} \mathbf{1}$; (b) $\mathrm{PhSeO}_{2} \mathrm{H}$ 2; (c-e) Polymers 4-6.

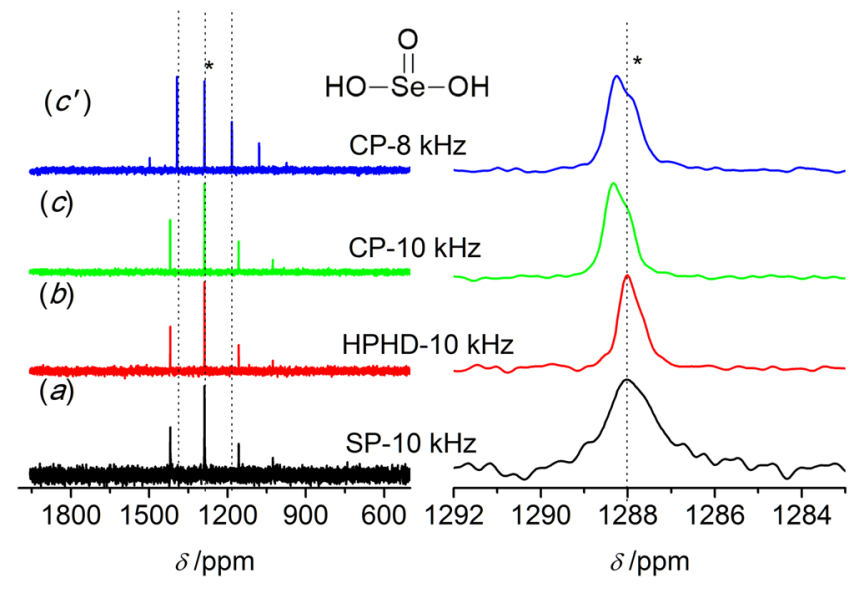

Figure 2. ${ }^{77} \mathrm{Se}$ MAS NMR spectra of $\mathrm{H}_{2} \mathrm{SeO}_{3} 1$ obtained by different pulse sequences. (a) Single pulse (SP); (b) High power ${ }^{1} \mathrm{H}$ decoupling (HPHD); (c, $\mathrm{c}^{\prime}$ ) Cross polarization (CP), 16 scans and the spinning speed of $10 \mathrm{kHz}$ or $8 \mathrm{kHz}$. The isotropic peak is labeled with an asterisk $(*)$.

etc $^{39-43}$. The method offers a beneficial solution to achieve higher SNR based on the applications of polarization transfer from the abundant proton $\left({ }^{1} \mathrm{H}\right)$ spin to low abundance nuclei i.e. ${ }^{77} \mathrm{Se}$ in the present paper via heteronuclear dipole-dipole interactions. The improvements are originated from enhanced sensitivity of the Se and faster longitudinal relaxation time $\left(T_{1}\right)$ of ${ }^{1} \mathrm{H}$ compared to ${ }^{77} \mathrm{Se}$ leading to shorter recycle delay time $\left(\mathrm{D}_{1}\right)$ between every acquisition $\left(\geq 5 T_{1}\right)^{44-46}$. Experiments through traditional method of SP and a single pulse sequence with special high power ${ }^{1} \mathrm{H}$ decoupling (HPHD) were also performed for comparison with the CP method. The CP method has been successfully applied to determine the state of the low-content selenium in several novel polymers. The novelty of the present paper is that the correlations of intensity of ${ }^{77} \mathrm{Se}$ NMR signal with the solid state and molecular structure of polymers were demonstrated for the first time. Herein, we wish to report our findings.

\section{Results}

Solid-state properties of the samples were initially investigated. Figure 1 presents the powder X-ray diffraction (PXRD) patterns of the model compounds and polymer materials containing ${ }^{77} \mathrm{Se}$. PXRD pattern of $\mathrm{H}_{2} \mathrm{SeO}_{3} 1$ is completely different from that of $\mathrm{PhSeO}_{2} \mathrm{H}$ 2, confirming their different crystal structures due to the presence of a benzene ring ${ }^{47}$. In contrast, all the polymer materials exhibit a broad reflection, indicating their amorphous structures ${ }^{48}$.

To optimize the experimental parameters for different pulse sequences, $\mathrm{H}_{2} \mathrm{SeO}_{3} \mathbf{1}$ was first measured and used as a secondary external reference standard. Figure 2 presents the ${ }^{77} \mathrm{Se}$ NMR spectra obtained by the different 


\begin{tabular}{|c|c|c|c|c|c|c|}
\hline Sample & Method & Number of scans & Delay time /s & Spinningspeed $/ \mathrm{kHz}$ & SNR & FWHM \\
\hline \multirow{4}{*}{$\mathrm{H}_{2} \mathrm{SeO}_{3}$} & SP & 16 & 45 & 10 & 13 & 101 \\
\hline & HPHD & 16 & 45 & 10 & 28 & 45 \\
\hline & $\mathrm{CP}$ & 16 & 30 & 10 & 45 & 51 \\
\hline & $\mathrm{CP}$ & 16 & 30 & 8 & 28 & 58 \\
\hline \multirow{4}{*}{$\mathrm{PhSeO}_{2} \mathrm{H}$} & SP & 1200 & 45 & 10 & 32 & 109 \\
\hline & HPHD & 1200 & 45 & 10 & 45 & 82 \\
\hline & $\mathrm{CP}$ & 1200 & 30 & 10 & 35 & 85 \\
\hline & $\mathrm{CP}$ & 1200 & 20 & 8 & 17 & 79 \\
\hline \multirow{4}{*}{ Polymer $4\left(-\mathrm{Se}_{-} \mathrm{CH}_{3}\right)$} & SP & 17408 & 5 & 10 & 4 & $\sim 1500$ \\
\hline & HPHD & 17408 & 5 & 10 & 5 & $\sim 1800$ \\
\hline & $\mathrm{CP}$ & 17408 & 5 & 10 & 13 & $\sim 1600$ \\
\hline & $\mathrm{CP}$ & 17108 & 5 & 13 & 16 & $\sim 1800$ \\
\hline Polymer 5 (-SeBr) & $\mathrm{CP}$ & 17408 & 5 & 10 & - & - \\
\hline \multirow{4}{*}{ Polymer $6\left(-\mathrm{SeO}_{3} \mathrm{H}\right)$} & SP & 17408 & 5 & 10 & 11 & 681 \\
\hline & HPHD & 17408 & 5 & 10 & 11 & 698 \\
\hline & $\mathrm{CP}$ & 17408 & 5 & 10 & 21 & 751 \\
\hline & $\mathrm{CP}$ & 17408 & 5 & 13 & 21 & 742 \\
\hline
\end{tabular}

Table 1. Experimental parameters and chemical shift for different compounds.

pulse sequencesas described in experimental section: single pulse (SP), high power ${ }^{1} \mathrm{H}$ decoupling (HPHD) and cross polarization (CP). To well illustrate the efficiency of the different methods, the intensity of the isotropic center-bands was normalized. A significant spinning side band manifold separated by the rotation frequency as a result of the chemical shift anisotropy (CSA) was observed in Fig. 2 left. The position of the isotropic center-bands (*) was determined by acquiring an additional spectrum at a different spinning speed of $8 \mathrm{kHz}$, which was enlarged in Fig. 2 right. The isotropic band remained at the same position for different speeds, while spinning side bands were changed (dashed lines, Fig. 2). Application of both HPHD and CP could enhance relative intensity of the resonance peak and narrow the lines (Fig. 2). An asymmetric isotropic band was observed for HPHD and CP, which might be related to defect in $\mathrm{H}_{2} \mathrm{SeO}_{3}$ crystal $^{49}$. The chemical shift obtained by SP sequence was chosen to be the reference standard (1288.1 ppm). Full Width at Half Maxima (FWHM) and the SNR of the isotropic band were obtained and listed in Table 1. The SNR was improved from 13 for SP to 28 for HPHD and to 45 for CP, and the corresponding FWHM decreased from $101 \mathrm{~Hz}$ to $45 \mathrm{~Hz}$ and to $51 \mathrm{~Hz}$. The existence of abundant ${ }^{1} \mathrm{H}$ would broaden the line of ${ }^{77} \mathrm{Se}$ spectra due to heteronuclear dipolar coupling between ${ }^{1} \mathrm{H}$ and ${ }^{77} \mathrm{Se}$, which could be removed by ${ }^{1} \mathrm{H}$ decoupling. Hence, the decoupling could improve SNR significantly and reduce FWHM, which explained the improvement for both HPHD and CP because of both of them including a decoupling pulse sequence. Moreover, the improvement for the $\mathrm{CP}$ was also related to the efficiency of polarization transfer between ${ }^{1} \mathrm{H}$ and ${ }^{77} \mathrm{Se}$. It should be mentioned that $\mathrm{CP}$ needed less time to achieve comparable results as HPHD due to the shorter $\mathrm{D}_{1}$ (Table 1 ).

A pureorganic compound, $\mathrm{PhSeO}_{2} \mathrm{H} 2$, was used to justify the efficiency of the three pulse sequences, and the spectra of 2 obtained were displayed in Fig. 3. As shown in Fig. 3 left, more spinning side bands were observed compared to $\mathrm{H}_{2} \mathrm{SeO}_{3}$ 1. The isotropic center-band was determined as marked by the asterisk (*), and its chemical shift was 1127.4 ppm with respect to $\mathrm{H}_{2} \mathrm{SeO}_{3}$ (Fig. 3 right). Similar to $\mathrm{H}_{2} \mathrm{SeO}_{3}, \mathrm{HPHD}$ and CP were able to enhance the relative intensity of the signal and narrow the line. The SNR was improved from 32 (SP) to 45 (HPHD) and to 35 (CP), and the FWHM was reduced from $109 \mathrm{~Hz}$ (SP) to $82 \mathrm{~Hz}$ (HPHD) and to $85 \mathrm{~Hz}$ (CP, Table 1). It was interesting that there was less enhancement of $\mathrm{CP}$ than that for $\mathrm{H}_{2} \mathrm{SeO}_{3}$. For $\mathrm{H}_{2} \mathrm{SeO}_{3}$, relatively strong hydrogen bond interactions induced the formation of well-ordered crystal and shortened the distance between Se atom and proton, whereas the presence of benzene ring in $\mathbf{2}$ distorted the hydrogen bond leading to total different crystal structure (Fig. 1) and consequently enlarged the distance between Se and proton ${ }^{50}$. These led to a weaker dipolar-dipolar interaction between ${ }^{77} \mathrm{Se}$ and ${ }^{1} \mathrm{H}$ spins for 2 , which explained the weaker enhancement of CP since the interaction was critical for cross polarization transfer efficiency. Moreover, low symmetry ${ }^{77} \mathrm{Se}$ environments would have a large anisotropic shielding, i.e.large CSA, which led to a large number of spinning side band ${ }^{51}$. Thus, the more spinning side bands for 2 might be attributed to lower symmetry ${ }^{77}$ Se environments compared to 1 as indicated from their molecular structures (Figs. 2 and 3 ).

The molar content of Se in polymer 6 (Scheme 1) was determined to be $1.46 \mathrm{mmol} / \mathrm{g}$ by an acid-base titration with $\mathrm{NaOH}$. If all the benzenes were selenized, the Se content should be $4.33 \mathrm{mmol} / \mathrm{g}$. Hence, only around $1 / 3$ of benzenes in the polymer were substituted (Scheme 1). Accordingly, similar low-content of Se could be found in polymer $4\left(-\mathrm{Se}-\mathrm{CH}_{3}\right)$, which lead to low sensitivity of ${ }^{77} \mathrm{Se}$ in NMR measurement. Figure 4 presents the spectra of polymer 4 obtained by the three methods. Several strong and wide spinning side bands could be observed in Fig. 4 left. The isotropic band (*) was determined by a faster speed acquisition $(13 \mathrm{kHz})$, and its chemical shift was around $198 \mathrm{ppm}$ (Fig. 4 right), close to values determined by solution $\mathrm{NMR}^{36,37}$. In contrast to compounds $\mathbf{1}$ and 2 (Figs 2 and 3), only CP was able to effectively improve the relative intensity of the signals with SNR changing from 4 to 13 (Fig. 4 and Table 1). No obvious change happened to the FWHM value for HPHD and CP compared to SP, which might indicate weaker heteronuclear coupling. Thus, the improvement by using $\mathrm{CP}$ was originated to shorter relaxation time of ${ }^{1} \mathrm{H}$ than ${ }^{77} \mathrm{Se}$ and the cross polarization transfer efficiency. As indicated from Table 1, the 


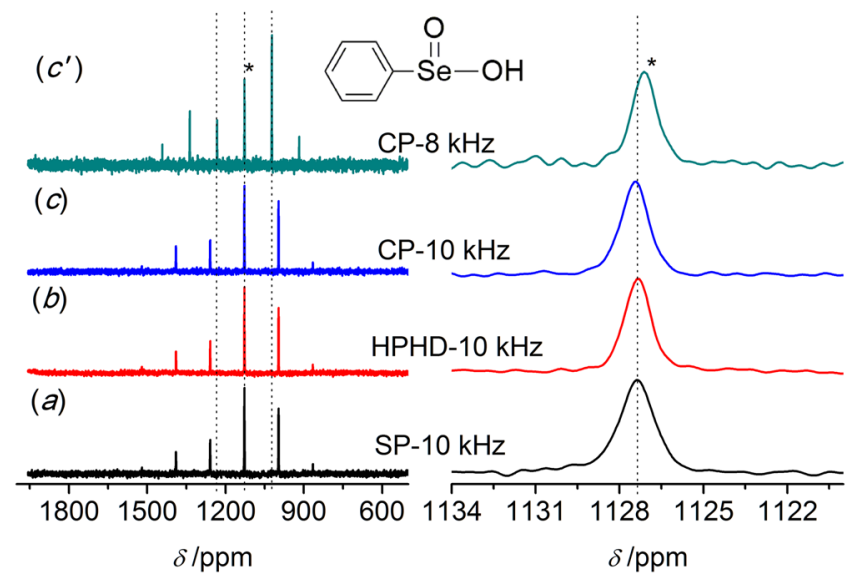

Figure 3. ${ }^{77} \mathrm{Se}$ MAS NMR spectra of $\mathrm{PhSeO}_{2} \mathrm{H} 2$ obtained by different pulse sequences. (a) Single pulse (SP); (b) High power ${ }^{1} \mathrm{H}$ decoupling (HPHD); (c, $\left.\mathbf{c}^{\prime}\right)$ Cross polarization (CP), 1200 scans and the spinning speed of $8 \mathrm{kHz}$ or $10 \mathrm{kHz}$. The isotropic peak is labeled with an asterisk (*).
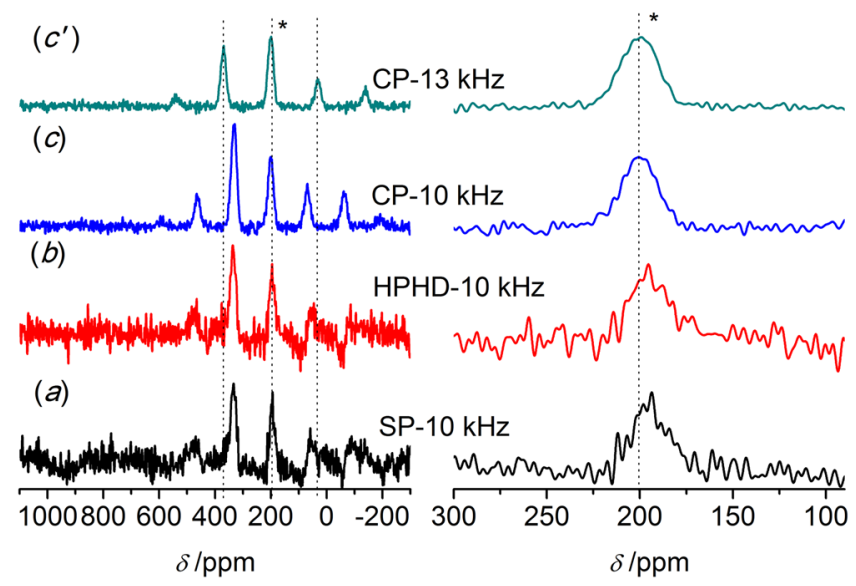

Figure 4. ${ }^{77} \mathrm{Se}$ MAS NMR spectra of polymer $4\left(-\mathrm{Se}_{-} \mathrm{CH}_{3}\right)$ obtained by different pulse sequences. (a) Single pulse (SP); (b) High power ${ }^{1} \mathrm{H}$ decoupling (HPHD); (c, $\left.\mathbf{c}^{\prime}\right)$ Cross polarization (CP), 17408 scans and the spinning speed of $10 \mathrm{kHz}$ or $13 \mathrm{kHz}$. The isotropic peak is labeled with an asterisk $\left(^{*}\right)$.

FWHM of the isotropic band became much broader than those of compounds $\mathbf{1}$ and 2, i.e. around $1600 \mathrm{~Hz}$. The broadening of the ${ }^{77}$ Se peak might be attributed to chemical shift distribution ${ }^{52}$. The chemical shift distribution might be originated from the change in solid-state from more ordered crystalline to amorphous structures ${ }^{38,47,48}$. This explanation can be well supported by PXRD results (Fig. 1).

In order to determine the state of Se in different polymers, and gain effect of different substituted functional groups on the spectrum quality, polymer $\mathbf{5}(-\mathrm{SeBr})$ was measured by SSNMR with the same experimental parameters applied for polymer 4 . Both ${ }^{79} \mathrm{Br}$ and ${ }^{81} \mathrm{Br}$ are half-integer $(\mathrm{I}=3 / 2)$ spins with large quadrupole moments $\left(3.3 \times 10^{-25}\right.$ and $2.8 \times 10^{-25} \mathrm{~cm}$, respectively), which were so large that yieldedextensive line broadening ${ }^{53}$. As a result, the sensitivity of Se in this polymer was too weak to give a reliable result under the same conditions as polymer 4 (Data not shown). As for the polymer 6 (- $\mathrm{SeO}_{3} \mathrm{H}$, Fig. 5), a well-defined single isotropic band and rather weak spinning side bands were observed. The isotropic band was determined to be $1023 \mathrm{ppm}$ in line with reports (Fig. 5 left, $*)^{47}$. Compared with polymer $4(\mathrm{ca} .1600 \mathrm{~Hz})$, the width of the isotropic bands became narrow $\left(c a .700 \mathrm{~Hz}\right.$, Table 1 ). The weak spinning side band might manifest higher symmetry ${ }^{77}$ Se environments in polymer 6 than that in polymer 4, leading to lower CSA ${ }^{51}$. Thus, the relative intensity of the isotropic band was much stronger than polymer 4showing higher SNReven for SP of 11 (Table 1). Although the polymers 4 and 6 were both in amorphous state (Fig. 1), the different line widths observed indicateddifferent motional properties and chemical shift distributions of Se atomas the substituted group changed from - $\mathrm{Se}-\mathrm{CH}_{3}$ to $-\mathrm{SeO}_{3} \mathrm{H}^{36,37}$. In addition, similar to polymer 4, only CP could significantly enhance the relative intensity of signals (Figure 5left), and correspondingly improved the SNR values from 11 to 21 (Table 1 ).

A comparison with the same spectrum range for polymer $\mathbf{4}$ and $\mathbf{6}$ was presented in Fig. 6 . No detectable signal assigned to polymer $4(a)$ could be observed in the spectrum of polymer $\mathbf{6}(b)$, indicating fully transformation 


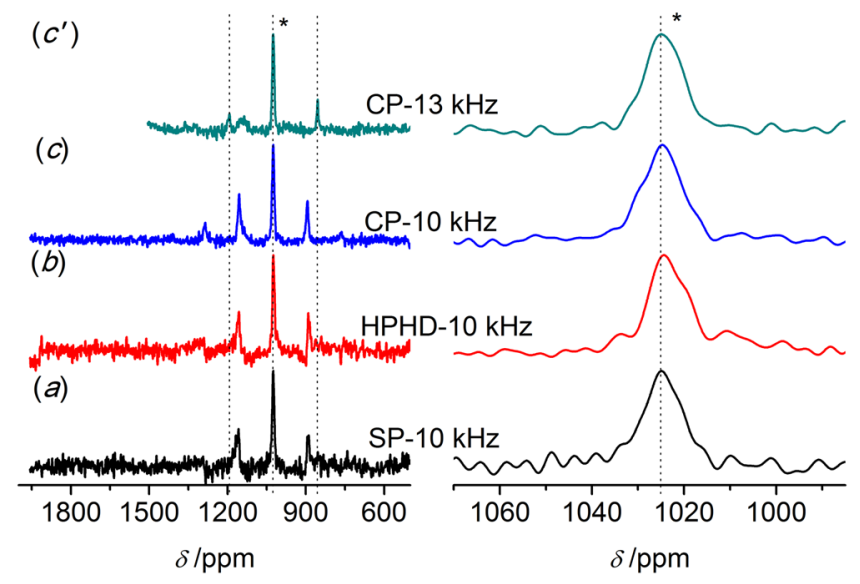

Figure 5. ${ }^{77} \mathrm{Se}$ MAS NMR spectra of polymer $6\left(-\mathrm{SeO}_{3} \mathrm{H}\right)$ obtained by different pulse sequences. (a) Single pulse(SP); (b) High power ${ }^{1} \mathrm{H}$ decoupling (HPHD); (c, $\mathbf{c}^{\prime}$ ) Cross polarization (CP), 17408 scans and the spinning speed of $10 \mathrm{kHz}$ or $13 \mathrm{kHz}$. The isotropic peak is labeled with an asterisk (*).

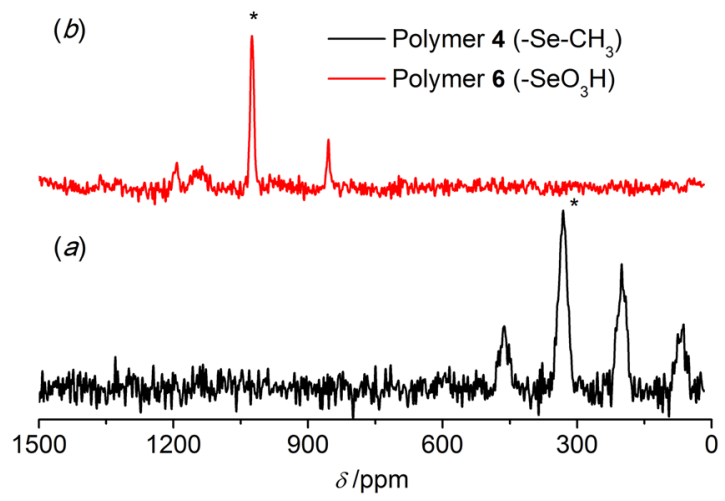

Figure 6. ${ }^{77} \mathrm{Se}$ CPMAS NMR spectra. (a) polymer $4\left(-\mathrm{Se}-\mathrm{CH}_{3}\right)$ and $(\mathbf{b})$ polymer $6\left(-\mathrm{SeO}_{3} \mathrm{H}\right), 17408$ scans and the spinning speed of $10 \mathrm{kHz}$ or $13 \mathrm{kHz}$. The isotropic peak is labeled with an asterisk $(*)$.

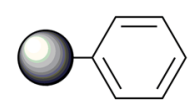

polystyrene 3

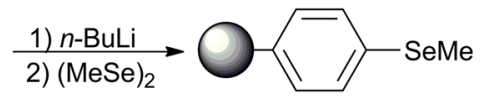

4

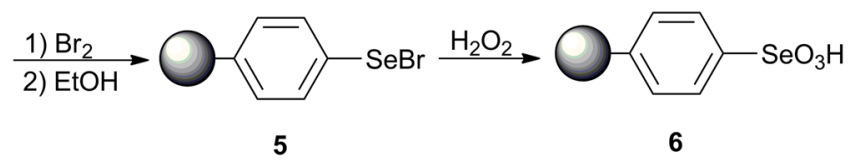

Figure 7. Preparation of the selenium-containing polymers 4-6.

from polymer 4 to polymer 6, which manifested that SSNMR could characterize the Se state in polymers even under low content.

\section{Conclusion}

The Se state in amorphous polymers even under low content was able to be efficiently determined by SSNMR through CP methods. It was found that low symmetry of molecular structure and less long range ordering structures of the solid would broaden the signal and enlarge the spinning side bands and conseqeuntly reduce SNR. These results would provide preliminary information for further investigation about the characterization of solidified selenium-containing materials from both molecular level perspective and macrostructure perspective. 


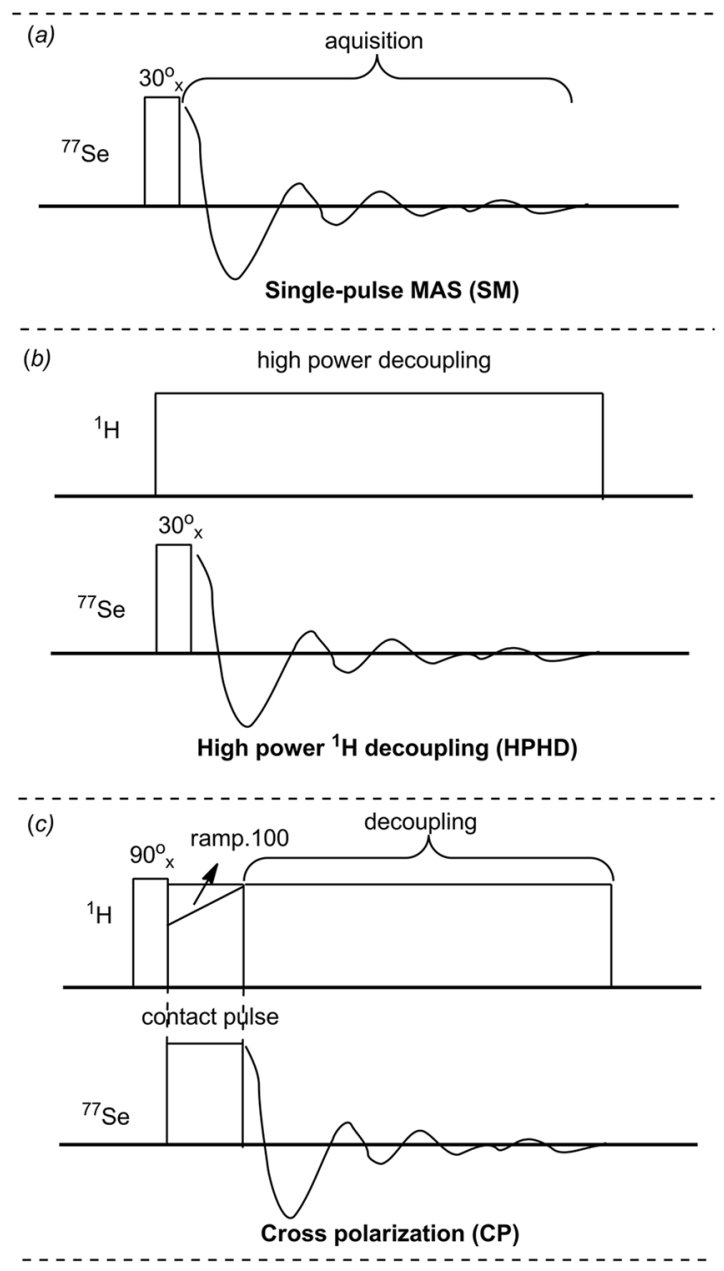

Figure 8. Schematic illustration of different pulse sequences. (a) Single pulse (SP); (b) High power ${ }^{1} \mathrm{H}$ decoupling (HPHD); (c) Cross polarization (CP).

\section{Methods}

Materials. Seleninic acid $\mathbf{1}\left(\mathrm{H}_{2} \mathrm{SeO}_{3}\right)$ and benzeneseleninic acid $\mathbf{2}\left(\mathrm{PhSeO}_{2} \mathrm{H}\right)$ were commercially available. The selenium-containing polymers 4-6 were prepared from polystyrene(Fig. 7).

Detailed procedures for the preparation of polymer 4 from polystyrene 3. $4.0 \mathrm{~g}$ of polystyrene resin 3 ( $1 \%$ cross-linked) was first immersed in cyclohexene $\left(30 \mathrm{~mL}\right.$ ) overnight under $\mathrm{N}_{2} .5 .6 \mathrm{~mL}$ of tetramethylethylenediamine (TMEDA, $37.2 \mathrm{mmol})$ and $48 \mathrm{mmol}$ of $n$-BuLi $(1.8 \mathrm{M}, 26.7 \mathrm{~mL})$ were added. The mixture was stirred at $65^{\circ} \mathrm{C}$ for $4 \mathrm{~h}$ and filtrated. The polymer was then washed by $10 \mathrm{~mL}$ of THF for three times and removed into a flask by washing with $40 \mathrm{~mL}$ of THF and mixed with $5 \mathrm{mmol}$ of $(\mathrm{MeSe})_{2}$ at $0^{\circ} \mathrm{C}$. After stirring $20 \mathrm{~min}$ at $0^{\circ} \mathrm{C}$, water $(3 \mathrm{~mL})$ was added and the color of the polymer turned yellow. The polymer was washed with THF (15 mL, twice), $\mathrm{H}_{2} \mathrm{O}\left(15 \mathrm{~mL}\right.$, twice), methanol (15 mL, twice), $\mathrm{CH}_{2} \mathrm{Cl}_{2}$ (15 mL, twice) and ether (15 mL, twice) subsequently and dried under vacuum at $65^{\circ} \mathrm{C}$ for $24 \mathrm{~h}$ to provide the polymer 4 .

Detailed procedures for the preparation of polymer 5 from 4 . $4.0 \mathrm{~g}$ of polymer $\mathbf{4}$ was immersed in chloroform $(60 \mathrm{~mL})$ under $\mathrm{N}_{2}$ overnight. A solution of $6 \mathrm{mmol}$ of $\mathrm{Br}_{2}$ in $\mathrm{CHCl}_{3}(20 \mathrm{~mL})$ was added and stirred for $30 \mathrm{~min}$ cooled with ice-water.The resin was then washed with anhydrous $\mathrm{EtOH}$ and removed into a flask containing $60 \mathrm{~mL}$ of anhydrous EtOH. The color turned red after heating at $70^{\circ} \mathrm{C}$ for $2 \mathrm{~h}$ and after filtration, the polymer was washed with $\mathrm{EtOH}(10 \mathrm{~mL}$, twice $)$ and $\mathrm{CH}_{2} \mathrm{Cl}_{2}(10 \mathrm{~mL}$, twice $)$ subsequently and then dried at $65^{\circ} \mathrm{C}$ under vacuum for $24 \mathrm{~h}$ to produce the polymer $5(4.3 \mathrm{~g})$.

Detailed procedures for the preparation of polymer $\mathbf{6}$ from 5 . $\quad 0.71 \mathrm{~g}$ of polymer $\mathbf{5}$ was immersed in $60 \mathrm{~mL} \mathrm{CHCl}_{3}$ under $\mathrm{N}_{2}$ overnight. $10 \mathrm{mmol}$ of $\mathrm{H}_{2} \mathrm{O}_{2}(30 \mathrm{w} / \mathrm{w} \%)$ was added at $0^{\circ} \mathrm{C}$. The color of the polymer turned white gradually after stirring at $25^{\circ} \mathrm{C}$ for $2 \mathrm{~h}$. After filtration, the resin was washed with EtOH $(10 \mathrm{~mL}$, twice) and $\mathrm{CH}_{2} \mathrm{Cl}_{2}\left(10 \mathrm{~mL}\right.$, twice) subsequently and then dried at $65^{\circ} \mathrm{C}$ under vacuum to produce the pure polymer $6(0.6849 \mathrm{~g})$. Acid-base titration with $\mathrm{NaOH}$ indicated that the content of Se in polymer 6 was $1.46 \mathrm{mmol} / \mathrm{g}$. 
Solid-state ${ }^{77}$ Se NMR. Solid-state ${ }^{77}$ Se NMR was performed on Bruker Avance III spectrometers operating magnetic field strengths of $9.4 \mathrm{~T}$, corresponding to Larmor frequencies at $76.3 \mathrm{MHz}$ for ${ }^{77}$ Se. A Bruker 4 mm double resonance HX MAS probe was used. $\mathrm{H}_{2} \mathrm{SeO}_{3}$ was used as a secondary external reference standard for $\mathrm{PhSeO}_{2} \mathrm{H}$ and polymers, and its isotropic chemical shift $(\delta)$ was calibrated to be $1288.1 \mathrm{ppm}$ with respect to dimethylselenide (0.0 ppm). Three pulse sequences were applied (Fig. 8): 1, a single pulse (SP). 2, a single pulse with high power ${ }^{1} \mathrm{H}$ decoupling (HPHD), and the composite pulse ${ }^{1} \mathrm{H}$ decoupling program is spinal64. The decoupling power was set to be $66.72 \mathrm{~W}$ with the pulse length of $9.0 \mu \mathrm{s} .3$, cross polarization $(\mathrm{CP})$ transfer from ${ }^{1} \mathrm{H}$ spin using optimized contact pulse durations of $4.5 \mathrm{~ms}$ or $5.0 \mathrm{~ms}$ (ramped for ${ }^{1} \mathrm{H}$ ), and two-pulse phase modulation (TPPM) ${ }^{1} \mathrm{H}$ decoupling during acquisition (spinal64). The decoupling power was set to be $66.72 \mathrm{~W}$ with the pulse length of $8.4 \mu \mathrm{s} .90^{\circ}$ radio frequency (rf) pulse length for ${ }^{1} \mathrm{H}$ excitation was $4.2 \mu \mathrm{s}$. In order to shorten recycle delay time $\left(D_{1}\right)$ between each acquisition, a $30^{\circ} \mathrm{rf}$ pulse of $1.54 \mu \mathrm{s}\left(\mathrm{D}_{1}, \mathrm{ca} .0 .1 T_{1}\right)$ was applied instead of the normally used $90^{\circ} \mathrm{rf}\left(\mathrm{D}_{1}, c a .5 T_{1}\right)$ for ${ }^{77}$ Se excitation in SP and HPHD pulse sequences. The applied $\mathrm{D}_{1}$ are listed in Table 1. The acquisition time for all of the three pulse sequences was set to be $4.56 \mathrm{~ms}$. Number of scans was varied from 16 to 17408 depending on the intensity of the samples. The data was acquired with spinning speed from $8 \mathrm{kHz}$ to $13 \mathrm{kHz}$. The experiment durations of compound $\mathrm{H}_{2} \mathrm{SeO}_{3}$ and $\mathrm{PhSeO}_{2} \mathrm{H}$ are $12 \sim 15 \mathrm{~min}$ and 10 15 h, respectively, and the duration for the polymers is $24 \mathrm{~h}$.

\section{References}

1. Wirth, T. Organoselenium chemistry. Vol. 208 (Springer, 2000).

2. Ogawa, A. In Main group metals in organic synthesis 813-866 (Wiley-VCH Verlag GmbH \& Co. KGaA, 2005).

3. Godoi, B., Schumacher, R. F. \& Zeni, G. Synthesis of heterocycles via electrophilic cyclization of alkynes containing heteroatom. Chem Rev 111, 2937-2980 (2011).

4. Wirth, T. In Organoselenium chemistryI-XIV (Wiley-VCH Verlag GmbH \& Co. KGaA, 2011).

5. Nomoto, A., Higuchi, Y., Kobiki, Y. \& Ogawa, A. Synthesis of selenium compounds by free radical addition based on visible-lightactivated se-se bond cleavage. Mini-Rev Med Chem 13, 814-823 (2013).

6. Weekley, C. M. \& Harris, H. H. Which form is that? The importance of selenium speciation and metabolism in the prevention and treatment of disease. Chem Soc Rev 42, 8870-8894 (2013).

7. Freudendahl, D. M., Santoro, S., Shahzad, S. A., Santi, C. \& Wirth, T. Green chemistry with selenium reagents: Development of efficient catalytic reactions. Angew Chem Int Ed 48, 8409-8411 (2009).

8. Santi, C., Santoro, S. \& Battistelli, B. Organoselenium compounds as catalysts in nature and laboratory. Curr Org Chem 14, $2442-2462(2010)$.

9. Santoro, S. et al. The green side of the moon: Ecofriendly aspects of organoselenium chemistry. RSC Adv 4, 31521-31535 (2014).

10. Breder, A. \& Ortgies, S. Recent developments in sulfur- and selenium-catalyzed oxidative and isohypsic functionalization reactions of alkenes. Tetrahedron Lett 56, 2843-2852 (2015).

11. Młochowski, J. \& Wójtowicz-Młochowska, H. Developments in synthetic application of selenium(iv) oxide and organoselenium compounds as oxygen donors and oxygen-transfer agents. Molecules 20, 10205-10243 (2015).

12. Ortgies, S., Depken, C. \& Breder, A. Oxidative allylic esterification of alkenes by cooperative selenium-catalysis using air as the sole oxidant. Org Lett 18, 2856-2859 (2016).

13. Guo, R., Huang, J., Huang, H. \& Zhao, X. Organoselenium-catalyzed synthesis of oxygen- and nitrogen-containing heterocycles. $\mathrm{Org}$ Lett 18, 504-507 (2016)

14. Cresswell, A. J., EeyStanley, T. C. \& Denmark, S. E. Catalytic, stereospecific syn-dichlorination of alkenes. Nat Chem 7, 146-152 (2015).

15. Luo, J., Zhu, Z., Liu, Y. \& Zhao, X. Diaryl selenide catalyzed vicinal trifluoromethylthioamination of alkenes. Org Lett 17, 3620-3623 (2015).

16. Deng, Z., Wei, J., Liao, L., Huang, H. \& Zhao, X. Organoselenium-catalyzed, hydroxy-controlled regio- and stereoselective amination of terminal alkenes: Efficient synthesis of 3-amino allylic alcohols. Org Lett 17, 1834-1837 (2015).

17. Ortgies, S. \& Breder, A. Selenium-catalyzed oxidative $\mathrm{C}\left(\mathrm{sp}^{2}\right)-\mathrm{H}$ amination of alkenes exemplified in the expedient synthesis of (aza-)indoles. Org Lett 17, 2748-2751 (2015).

18. Chen, F., Tan, C. K. \& Yeung, Y.-Y. C2-symmetric cyclic selenium-catalyzed enantioselective bromoaminocyclization. J Am Chem Soc 135, 1232-1235 (2013).

19. Trenner, J., Depken, C., Weber, T. \& Breder, A. Direct oxidative allylic and vinylic amination of alkenes through selenium catalysis. Angew Chem IntEd 52, 8952-8956 (2013).

20. Sancineto, L. et al. Selenium catalyzed oxidation of aldehydes: Green synthesis of carboxylic acids and esters. Molecules 20, 10496-10510 (2015).

21. Santi, C., Di Lorenzo, R., Tidei, C., Bagnoli, L. \& Wirth, T. Stereoselective selenium catalyzed dihydroxylation and hydroxymethoxylation of alkenes. Tetrahedron 68, 10530-10535 (2012).

22. Santoro, S., Santi, C., Sabatini, M., Testaferri, L. \& Tiecco, M. Eco-friendly olefin dihydroxylation catalyzed by diphenyl diselenide. Adv Synth Catal 350, 2881-2884 (2008).

23. ten Brink, G.-J., Fernandes, B. C. M., van Vliet, M. C. A., Arends, I. W. C. E. \& Sheldon, R. A. Selenium catalysed oxidations with aqueous hydrogen peroxide. Part i: Epoxidation reactions in homogeneous solution. J Chem Soc, Perkin Trans 1, 224-228 (2001).

24. Jing, X., Yuan, D. \& Yu, L. Green and practical oxidative deoximation of oximes to ketones or aldehydes with hydrogen peroxide/air by organoselenium catalysis. Adv Synth Catal 359, 1194-1201 (2017).

25. Wang, Y., Yu, L., Zhu, B. \& Yu, L. Design and preparation of a polymer resin-supported organoselenium catalyst with industrial potential. J Mater Chem A 4, 10828-10833 (2016).

26. Yu, L. et al. Facile synthesis of 2-methylenecyclobutanones via $\mathrm{Ca}(\mathrm{OH})_{2}$-catalyzed direct condensation of cyclobutanone with aldehydes and $(\mathrm{PhSe})_{2}$-catalyzed baeyer-villiger oxidation to 4-methylenebutanolides. Green Chem 16, 287-293 (2014).

27. Yu, L., Wang, J., Chen, T., Wang, Y. \& Xu, Q. Recyclable 1,2-bis[3,5-bis(trifluoromethyl)phenyl]diselane-catalyzed oxidation of cyclohexene with $\mathrm{H}_{2} \mathrm{O}_{2}$ : A practical access to trans-1,2-cyclohexanediol. Appl Organomet Chem 28, 652-656 (2014).

28. Yu, L. et al. Organoselenium-catalyzed selectivity-switchable oxidation of $\beta$-ionone. Catal Sci Technol 6, 1804-1809 (2016).

29. Yu, L., Chen, F. \& Ding, Y. Organoselenium-catalyzed oxidative ring expansion of methylenecyclopropanes with hydrogen peroxide. ChemCatChem 8, 1033-1037 (2016).

30. Yu, L., Ye, J., Zhang, X., Ding, Y. \& Xu, Q. Recyclable (PhSe) $)_{2}$-catalyzed selective oxidation of isatin by $\mathrm{H}_{2} \mathrm{O}_{2}$ : A practical and wastefree access to isatoic anhydride under mild and neutral conditions. Catal Sci Technol 5, 4830-4838 (2015).

31. Zhang, X. et al. Organoselenium-catalyzed Baeyer-Villiger oxidation of $\alpha, \beta$-unsaturated ketones by hydrogen peroxide to access vinyl esters. Adv Synth Catal 357, 955-960 (2015).

32. Zhang, X., Sun, J., Ding, Y. \& Yu, L. Dehydration of aldoximes using PhSe(O)OH as the pre-catalyst in air. Org Lett 17, 5840-5842 (2015). 
33. Yu, L. et al. Organoselenium-catalyzed mild dehydration of aldoximes: An unexpected practical method for organonitrile synthesis. Org Lett 16, 1346-1349 (2014).

34. Yu, L., Wang, J., Chen, T., Ding, K. \& Pan, Y. Access to cyclohexane-1,2-diol through the diphenyldiselenide catalyzed oxidation of cyclohexene by hydrogen peroxide. Chin J Org Chem 33, 1096-1099 (2013).

35. Rayman, M. P. Selenium and human health. Lancet 379, 1256-1268 (2012).

36. Duddeck, H. Selenium-77 nuclear magnetic resonance spectroscopy. Prog Nucl Mag Res Sp 27, 1-323 (1995).

37. Demko, B. A. \& Wasylishen, R. E. Solid-state selenium-77 NMR. Prog Nucl Mag Res Sp 54, 208-238 (2009).

38. Sen, S., Kaseman, D. C., Hung, I. \& Gan, Z. ${ }^{77}$ se nuclear spin-lattice relaxation in binary ge-se glasses: Insights into floppy versus rigid behavior of structural units. J Phys Chem B 119, 5747-5753 (2015).

39. Pham, T. N. et al. Analysis of amorphous solid dispersions using $2 \mathrm{~d}$ solid-state $\mathrm{nmr}$ and ${ }^{\mathrm{h}} \mathrm{h} \mathrm{t}_{1}$ relaxation measurements. Mol Pharm 7, 1667-1691 (2010).

40. Kinage, A. K., Prashar, A. K., Berlier, G. \& Chaturvedi, R. K. Growth of hydrothermally stable meso-porous silica structure interconnected around micro-porous zeolite crystals. Mater Charact 62, 1166-1172 (2011).

41. Miksa, B. et al. Application of ionic liquid matrices in spectral analysis of poly(lactide)-solid state NMR spectroscopy versus matrixassisted laser desorption/ionization time-of-flight (maldi-tof) mass spectrometry. Anal Methods 4, 377-383 (2012).

42. Major, Y. et al. Towards the rationalization of the maldi process: A combined mass spectrometry/solid-state nmr approach. Anal Methods 4, 3118-3126 (2012).

43. Zhu, X., Liu, B., Zheng, S. \& Gao, Y. Quantitative and structure analysis of pectin in tobacco by $13 \mathrm{c} \mathrm{cp} / \mathrm{mas} \mathrm{nmr}$ spectroscopy. Anal Methods 6, 6407-6413 (2014).

44. Reyes-Garcia, E. A., Sun, Y., Reyes-Gil, K. \& Raftery, D. ${ }^{15} \mathrm{~N}$ solid state NMR and EPR characterization of N-doped $\mathrm{TiO}_{2}$ photocatalysts. J Phys Chem C 111, 2738-2748 (2007).

45. Zujovic, Z. D., Zhang, L., Bowmaker, G. A., Kilmartin, P. A. \& Travas-Sejdic, J. Self-assembled, nanostructured aniline oxidation products: A structural investigation. Macromolecules 41, 3125-3135 (2008).

46. Potrzebowski, M. J., Katarzyński, R. \& Ciesielski, W. Selenium-77 and Carbon-13 high-resolution solid-state NMR studies of selenomethionine. Magn Reson Chem 37, 173-181 (1999).

47. Kolmas, J., Kuras, M., Oledzka, E. \& Sobczak, M. A solid-state NMR study of selenium substitution into nanocrystalline hydroxyapatite. Int J Mol Sci 16, 11452-11464 (2015).

48. Vogt, F. G. \& Williams, G. R. Analysis of a nanocrystalline polymer dispersion of ebselen using solid-state nmr, raman microscopy, and powder X-ray diffraction. Pharm Res 29, 1866-1881 (2012).

49. Bureau, B. et al. Solid state ${ }^{77}$ Se NMR investigations on arsenic-selenium glasses and crystals. Solid State Sci 5, 219-224 (2003).

50. Powell, J., Kalakewich, K., Uribe-Romo, F. J. \& Harper, J. K. Solid-state NMR and DFT predictions of differences in cooh hydrogen bonding in odd and even numbered N-alkyl fatty acids. Phys Chem Chem Phys 18, 12541-12549 (2016).

51. Struppe, J., Zhang, Y. \& Rozovsky, S. ${ }^{77}$ Se chemical shift tensor of l-selenocystine: Experimental NMR measurements and quantum chemical investigations of structural effects. J Phys Chem B 119, 3643-3650 (2015).

52. Duer, M. J. In Solid-state nmr spectroscopy principles and applications.237-279 (Blackwell Science Ltd, 2007).

53. Taylor, R. E. Setting up ${ }^{13}$ CCP/MAS experiments. Concepts Magn Reson Part A 22A, 37-49 (2004).

\section{Acknowledgements}

This work was supported by the National Nature Science Foundation of China (No. 21603186, 21202141), Priority Academic Program Development (PAPD) of Jiangsu Higher Education Institutions, the High Level Talent Support Project of Yangzhou University, the Initial Scientific Research Fund of Yangzhou University (137010931).

\section{Author Contributions}

D. Wei and L. Yu supervised the overall project and performed some of the experiments. MT Han performed some of the experiments.

\section{Additional Information}

Competing Interests: The authors declare that they have no competing interests.

Publisher's note: Springer Nature remains neutral with regard to jurisdictional claims in published maps and institutional affiliations.

Open Access This article is licensed under a Creative Commons Attribution 4.0 International License, which permits use, sharing, adaptation, distribution and reproduction in any medium or format, as long as you give appropriate credit to the original author(s) and the source, provide a link to the Creative Commons license, and indicate if changes were made. The images or other third party material in this article are included in the article's Creative Commons license, unless indicated otherwise in a credit line to the material. If material is not included in the article's Creative Commons license and your intended use is not permitted by statutory regulation or exceeds the permitted use, you will need to obtain permission directly from the copyright holder. To view a copy of this license, visit http://creativecommons.org/licenses/by/4.0/.

(C) The Author(s) 2017 